Check for updates

Cite this: RSC Adv., 2019, 9, 20513

Received 28th May 2019

Accepted 27th June 2019

DOI: $10.1039 / c 9 r a 04034 j$

rsc.li/rsc-advances

\title{
pH/redox sensitive nanoparticles with platinum(Iv) prodrugs and doxorubicin enhance chemotherapy in ovarian cancer
}

\author{
Guyu Zhang, ${ }^{\text {a }}$ Yimin Zhu, ${ }^{\text {b }}$ Yushu Wang, ${ }^{\mathrm{c}}$ Dengshuai Wei, ${ }^{\text {de }}$ Yixin $\mathrm{Wu},{ }^{\mathrm{f}}$ \\ Liuchun Zheng, (D) de Huimin Bai, ${ }^{* a}$ Haihua Xiao*de and Zhenyu Zhang (D) *a
}

\begin{abstract}
$\mathrm{pH} /$ redox sensitive, dual drug loaded nanoparticles were prepared from poly(ethylene glycol)-blockpoly(L-lysine) (PEG-b-PLL) for improving cancer therapy. Platinum(IV) and cis-aconitic anhydridedoxorubicin (CAD) were anchored to lysine residual amine groups of PLL to form polymer prodrug conjugates, which then self-assembled into nanoparticles with hydrophobic platinum(IV) prodrugs and $\mathrm{CAD}$ as the core. The nanoparticles were stable in neutral environments, but once under acidic and reductive conditions, the drugs were rapidly released. The dual-loaded nanoparticles had comparable intracellular toxicity to the regimen of combined application of free cisplatin and doxorubicin.
\end{abstract}

\section{Introduction}

Ovarian cancer is one of the most lethal gynaecological cancers with 22530 new cases and 13980 associated mortalities reported in 2018. ${ }^{1}$ Combination chemotherapy of cisplatin (Pt) and doxorubicin (Dox) has been widely used as an anti-cancer regimen. ${ }^{2,3}$ In clinical trials, Pt and Dox were combined for recurrent ovarian cancer. ${ }^{4}$ However, the high toxicity of Dox and drug resistance against Pt caused by glutathione (GSH) compromised the therapy outcomes. ${ }^{5-7}$ One possible way to overcome these obstacles is to modify prodrugs with specific linkages cleaved under the intracellular environment of tumors, such as low $\mathrm{pH}^{8}$ and abundant glutathione, ${ }^{9}$ inducing cytoplasmic drug release. A Pt(Iv) prodrug was considered as a promising agent to overcome platinum resistance. Xiao et al. reported that inert $\mathrm{Pt}(\mathrm{Iv})$ prodrugs could transform into toxic $\mathrm{Pt}(\mathrm{II})$ through abundant intracellular GSH and then kill the

\footnotetext{
${ }^{a}$ Beijing Chaoyang Hospital Affiliated to Capital Medical University, Department of Gynaecological and Obstetric, Beijing 100000, P. R. China. E-mail: zhenyuzhang2000@163.com

${ }^{b}$ Affiliated Guangdong Medical University, Department of Oncology, Zhanjiang 524000, P. R. China

'Key Laboratory of Bio-based Material Science and Technology Ministry of Education, Northeast Forestry University, Harbin 150040, P. R. China

${ }^{d}$ Beijing National Laboratory for Molecular Sciences, State Key Laboratory of Polymer Physics and Chemistry, Institute of Chemistry, Chinese Academy of Sciences, Beijing 100190, P. R. China. E-mail: hhxiao@iccas.ac.cn

${ }^{e}$ University of Chinese Academy of Sciences, Beijing 100049, P. R. China

${ }^{f}$ College of Life Science and Technology, Beijing University of Chemical Technology, Beijing 100029, P. R. China
}

$\dagger$ Electronic supplementary information (ESI) available. See DOI: $10.1039 / \mathrm{c} 9 \mathrm{ra} 04034 \mathrm{j}$ cancer cells. ${ }^{10,11}$ Herein, we developed a Pt(Iv) prodrug with a long lipid chain $\left(\mathrm{C}_{16}\right)$ and carboxyl group in the axial positions (Scheme S1†). CAD has also been proved to exhibit pH sensitive features $^{12}$ (Scheme S2 $\dagger$ ). Poly(ethylene glycol)-block-poly(Llysine) (PEG- $b$-PLL) has been widely used as polymer drug carrier due to its superior biocompatibility and stability. ${ }^{13,14}$ Hence, both Pt(Iv) and CAD could be coupled with the amine groups of PLL block via amidation to self-assemble into nanoparticle (NPs (Pt(Iv) + CAD)) (Scheme S3†). Prodrug nanoparticles emerged as promising candidates to deliver multiple anti-cancer agents. ${ }^{15-17}$ Compared with conventional chemotherapy, these nanometer-sized drugs exhibited considerable advantages, such as passive enhanced permeability and retention (EPR) impact on tumors, improved stability and solubility as well as lower toxicity in the bloodstream. ${ }^{18-20}$ When the $\mathrm{pH} /$ redox dual sensitive nanoparticles were internalized by tumor cells, Pt(Iv) and CAD could be rapidly released under the acidic environment of tumor cells, and then turned into Pt(II) and Dox to kill tumor cells. Robust cell-killing effect on ovarian cancer cells could be achieved with NPs (Pt(Iv) + CAD) through synergistic effects (Scheme 1). Additionally, the $\mathrm{pH} /$ redox sensitive bonds of $\mathrm{Pt}(\mathrm{Iv})$ prodrugs and $\mathrm{CAD}$ limited non-specific drug release in normal cells and alleviated the non-specific cell killing.

\section{Results}

2.1. Synthesis and characterization of $\mathrm{pH} /$ redox sensitive codelivery nanoparticles

In this study, we prepared a biocompatible $\mathrm{pH} /$ redox sensitive nanoparticle for co-delivery of CAD and $\mathrm{Pt}(\mathrm{Iv})$ prodrugs. The $\mathrm{CAD}$ or $\mathrm{Pt}(\mathrm{Iv})$ were conjugated with the amine groups of PLL via 


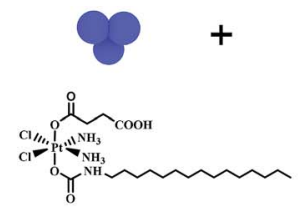

Pt(IV)
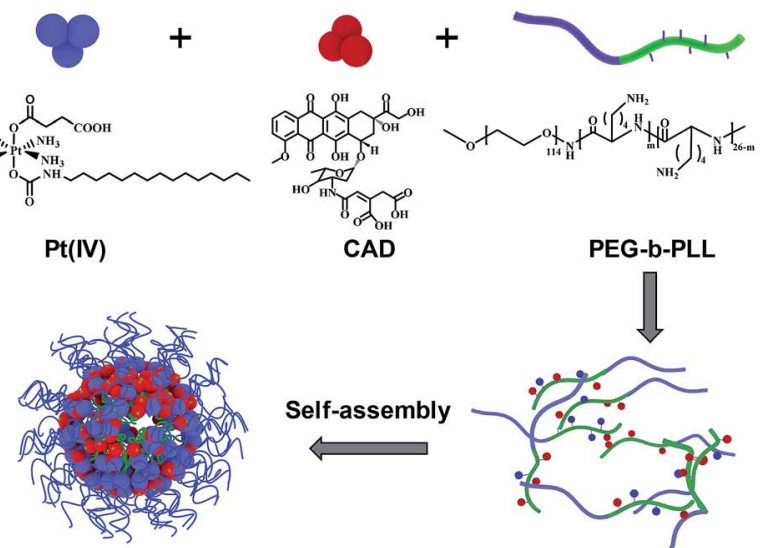

Dual drug loaded nanoparticle

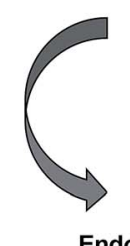

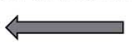

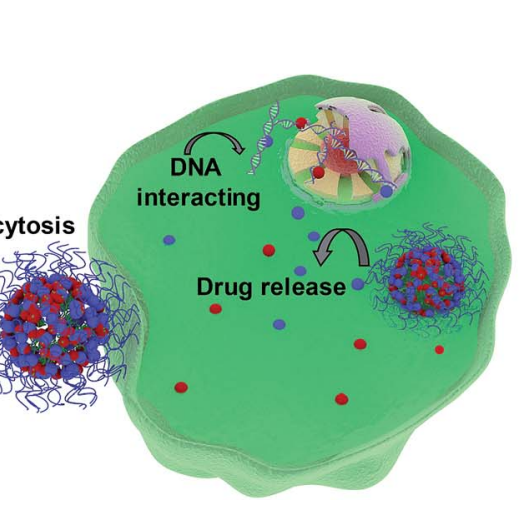

Self-assembly

Scheme 1 Preparation process of NPs (Pt(IV) + CAD) and the cell killing mechanism

1-(3-dimethylaminopropyl)-3-ethyl carbodiimide hydrochloride (EDC) and $N$-hydroxysuccinimide; 1-hydroxypyrrolidine-2,5dione (NHS) mediated amidation reaction (Schemes $\mathrm{S} 4$ and S5 $\dagger$ ). The maximum Pt loading (8\%) was achieved when drug to polymer molar reached $8: 1$, the optimal Dox loading was $10 \%$ when drug to polymer molar ratio was at $6: 1$ (Fig. S1 $\dagger$ ). After a series of optimizations, drugs to polymer molar ratio (Pt : Dox : PEG- $b$-PLL) of $8: 6: 1$ was selected as the optimized ratio to synthesize the NPs (Pt(Iv) + CAD) in the following study. Eventually, the NPs (Pt(Iv) + CAD) with Pt to Dox ratio at $2: 1$ were obtained. All the synthesized prodrugs and copolymers were confirmed by using ${ }^{1} \mathrm{H}-\mathrm{NMR}$ (Fig. S4-S6†). Furthermore, the NPs (Pt(Iv) + CAD) showed great size stability under PBS and $10 \%$ fetal bovine serum (Fig. 1). The size and PDI of nanoparticles are summarized in Table 1, suggesting the nanoparticles have been prepared.

\subsection{Cellular uptake of platinum and Dox and intracellular drug localization}

The uptake of platinum by A2780 and A2780DDP (both cell lines were obtained from the Medical Department of Jilin University in China) was qualified by ICP-MS after incubation with cells for $2 \mathrm{~h}$ and $7 \mathrm{~h}$. As shown in Fig. 2, the process of cell uptake was time dependent. The result shows that the amount of platinum endocytosis for NPs (Pt(Iv) + CAD) and NPs (Pt(Iv)) at $7 \mathrm{~h}$ were 3.5

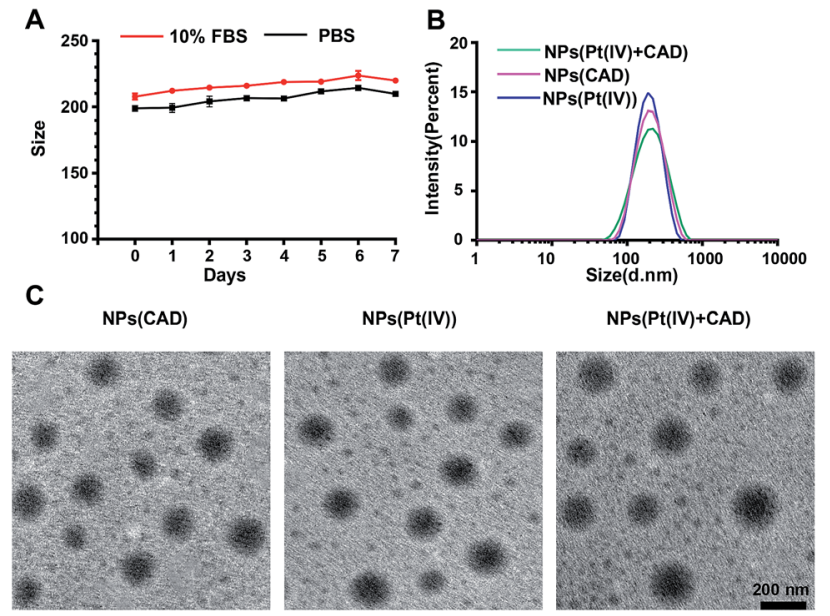

Fig. 1 Characterization of nanoparticles. Stability of NPs (Pt(Iv) + CAD) (A). DLS of nanoparticles (B) and TEM images of nanoparticles (C). Scale bar: $200 \mathrm{~nm}$.

to 4 times more than free Pt on A2780 and A2780DDP. No obvious difference was observed between NPs (Pt(Iv) + CAD) and NPs (Pt(Iv)). Flow cytometry was here applied to monitor the internalization of Dox after $7 \mathrm{~h}$ treatment. Compared with free Dox, the fluorescence of nanoparticles group shifted right significantly. Up to three times enhancement of intercellular uptake was achieved on nanoparticles group. To further visualize the endocytosis pathway of nanoparticles, confocal laser scanning microscopy (CLSM) was applied to locate the nanoparticles. In Fig. 3, after incubation with free Dox and nanoparticles for $2 \mathrm{~h}$ and $7 \mathrm{~h}$ respectively, red signal was mainly presented in the nucleus of A2780DDP. The uptake pattern of nanoparticles was in a time dependent manner as the red fluorescence intensity of $7 \mathrm{~h}$ incubation was brighter than $2 \mathrm{~h}$ in all groups, which was consistent with the result of uptake via ICP-MS shown in Fig. 2. Meanwhile, the red fluorescence signal of nanoparticles group was stronger than free Dox, suggesting the nanoparticles could facilitate the internalization process of prodrug.

\section{3. $\mathrm{pH} /$ redox responsive drug release}

To investigated the triggered release property of nanoparticles, cumulative platinum and Dox release in vitro from nanoparticles were observed at $\mathrm{pH} 7.4, \mathrm{pH} 5.0, \mathrm{pH} 4.0$ and in $10 \mathrm{mmol}$ GSH solutions, respectively. At $\mathrm{pH} 7.4$, which was similar to $\mathrm{pH}$ value of normal tissue and circulatory system, the platinum release is less than $20 \%$ up to $40 \mathrm{~h}$, while in the

Table 1 The size and PDI of NPs (Pt(IV) + CAD), NPs (Pt(IV)) and NPs (CAD)

\begin{tabular}{lll}
\hline & Particle size (nm) & PDI \\
\hline NPs (Pt(Iv) + CAD) & $198.6 \pm 14.0$ & 0.21 \\
NPs (Pt(Iv)) & $194.5 \pm 10.1$ & 0.2 \\
NPs (CAD) & $185.7 \pm 7.1$ & 0.18
\end{tabular}



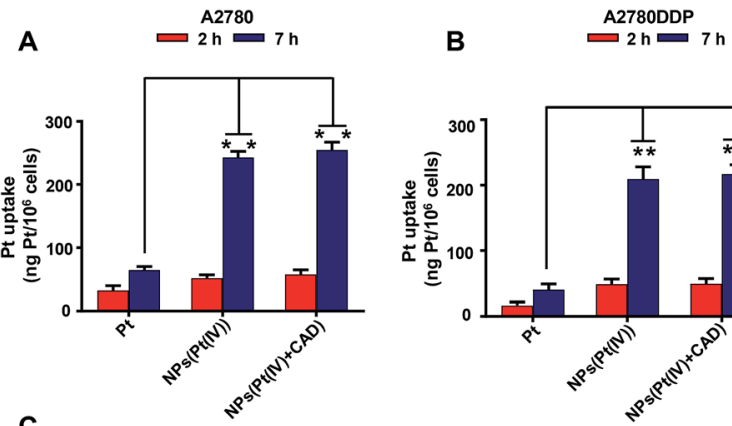

C

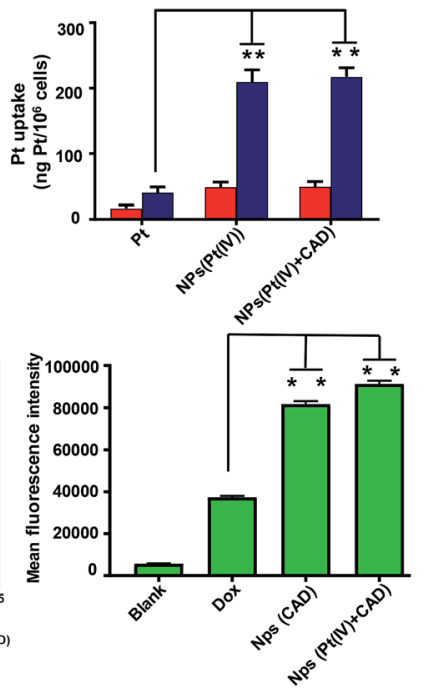

Fig. 2 In vitro cellular uptake of platinum and doxorubicin. Quantification of the platinum uptake on A2780 (A), A2780DDP (B) by ICP-MS. Quantification of the Dox uptake on A2780DDP by flow cytometry (C). Significance is defined as $* * P<0.001, * P<0.01$.

reductive environment such as in GSH solution, platinum release rate accelerated significantly and reached almost 90\% after incubation for $48 \mathrm{~h}$. Correspondingly, the Dox release was almost retarded under neutral environment, the cumulative release rate was $20 \%$ after incubation for $96 \mathrm{~h}$. However, at $\mathrm{pH}$ 5.0 and 4.0 , the release rate of Dox was elevated as the $\mathrm{pH}$ decreased. The cumulative Dox release rate ultimately increased to $40 \%$ and $50 \%$, respectively. This result indicated that acidic and reductive environment enhanced the cleavage of the amide bond between CAD prodrugs and PLL and induced Dox and Pt release (Fig. 4).

\subsection{In vitro cytotoxicity}

The cytotoxicity of Pt, Dox, Pt + Dox, NPs (Pt(Iv)), NPs (CAD) and NPs (Pt(Iv) + CAD) were evaluated by MTT assay on A2780 cells and Pt resistant cells A2780DDP. In Fig. 5, NPs (Pt(IV)) significantly increased cytotoxicity $\left(\mathrm{IC}_{50}=3.80 \mu \mathrm{M}, \mathrm{IC}_{50}=9.61 \mu \mathrm{M}\right)$ compared to free $\mathrm{Pt}\left(\mathrm{IC}_{50}=9.41 \mu \mathrm{M}, \mathrm{IC}_{50}=31.03 \mu \mathrm{M}\right)$ to $\mathrm{A} 2780$ and A2780DDP. However, the NPs (CAD) $\left(\mathrm{IC}_{50}=3.84 \mu \mathrm{M}, \mathrm{IC}_{50}=\right.$ $5.7 \mu \mathrm{M})$ manifested decreased drug potency compared to free Dox $\left(\mathrm{IC}_{50}=1.08 \mu \mathrm{M}, \mathrm{IC}_{50}=1.10 \mu \mathrm{M}\right)$, suggesting NPs $(\mathrm{CAD})$ could attenuate the toxicity. For the combination of free Pt and Dox $\left(\mathrm{IC}_{50}=0.75 \mu \mathrm{M}, \mathrm{IC}_{50}=0.61 \mu \mathrm{M}\right)$ and the NPs (Pt(Iv) $\left.+\mathrm{CAD}\right)$ $\left(\mathrm{IC}_{50}=0.72 \mu \mathrm{M}, \mathrm{IC}_{50}=0.60 \mu \mathrm{M}\right)$, they almost exhibited similar toxicity against ovarian cancer cells. The Combination Index (CI) was applied here to assess the synergistic effect of the NPs $(\mathrm{Pt}(\mathrm{Iv})+\mathrm{CAD})$ as well as Pt + Dox, the CI values of higher than, equal to, and lower than 1 represented antagonism, additivity, and synergism, respectively. ${ }^{21}$ The $\mathrm{IC}_{50}$ value of each group and CI of NPs (Pt(IV) + CAD) as well as Pt + Dox were summarized in Table 2, showing robust synergy effects for NPs (Pt(Iv) + CAD)

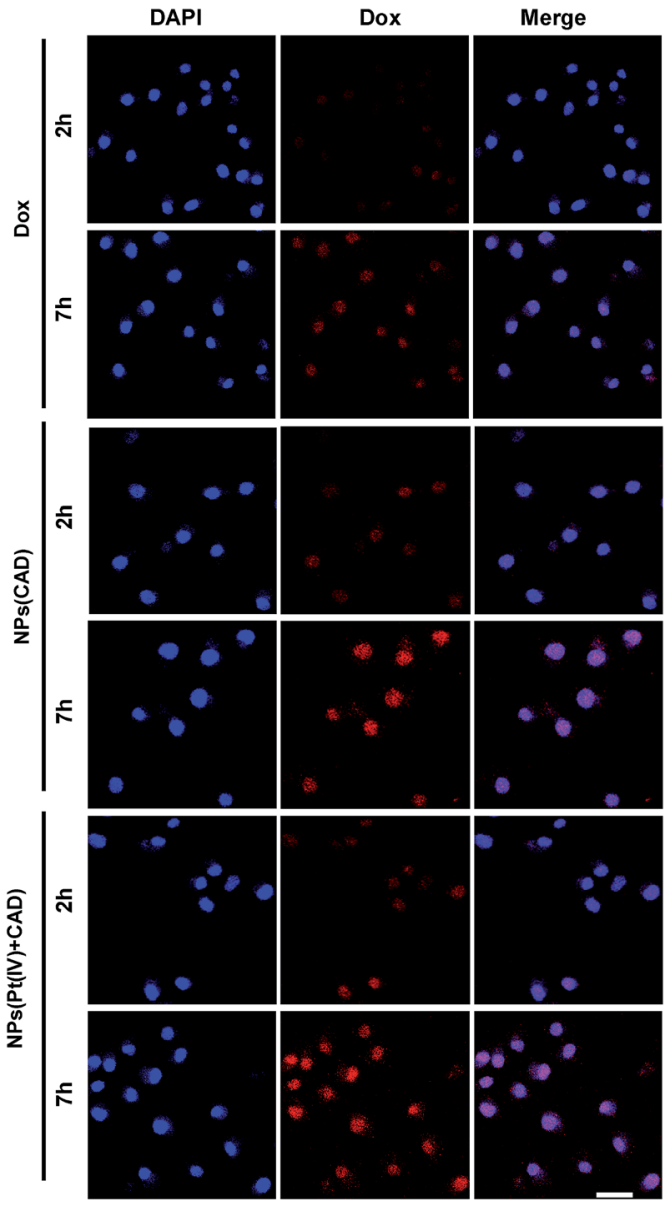

Fig. 3 CLSM images of A2780DDP incubated with free Dox and nanoparticles for $2 \mathrm{~h}$ and $7 \mathrm{~h}$. The blue fluorescence comes from a nuclear dye DAPI. The red fluorescence comes from Dox. Scale bar: $20 \mu \mathrm{m}$.

that were achieved in $\mathrm{A} 2780$ and $\mathrm{A} 2780 \mathrm{DDP}$ cell line $\left(\mathrm{CI}_{50}=\right.$ $0.55, \mathrm{CI}_{50}=0.21$ ).

\subsection{Cell cycle and apoptosis analysis}

To clarify the mechanism of cell death induced by NPs (Pt(Iv) + CAD), the cell cycle and apoptosis analysis were carried out via flow cytometry. During the normal cell cycle, $\mathrm{G}_{1}$ phase initiated

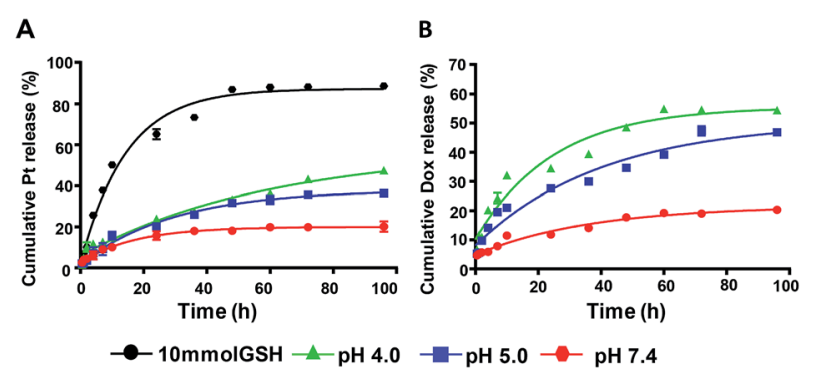

Fig. 4 The drug release profile of NPs (Pt(IV) + CAD). The platinum release under different conditions $(A)$, the Dox release under different conditions (B). 

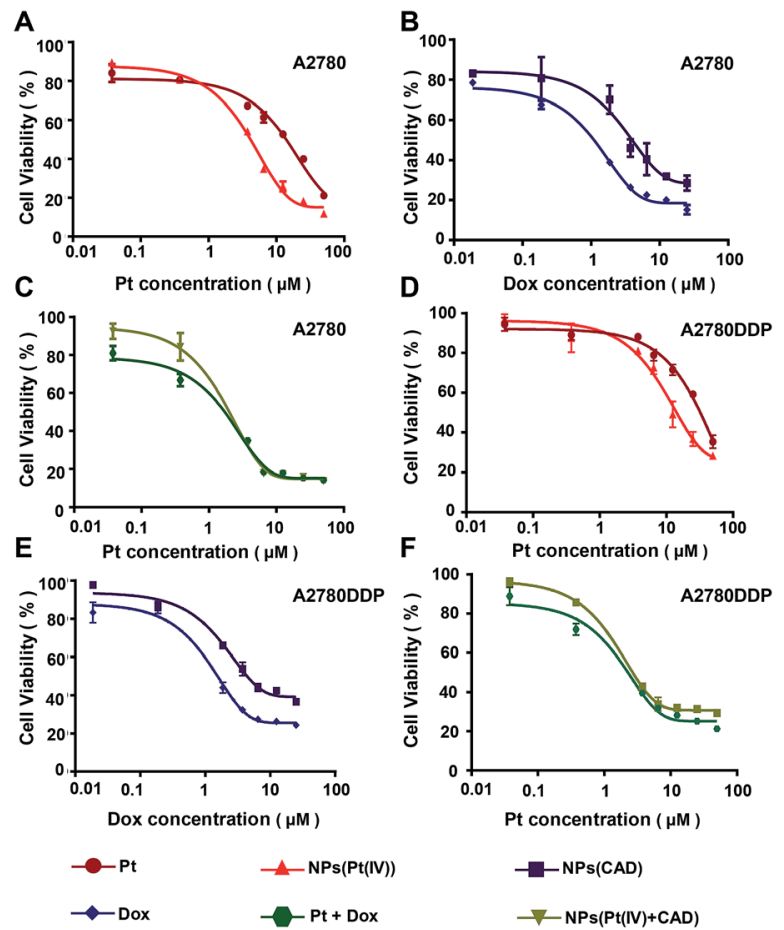

Fig. 5 In vitro evaluation of anticancer activity of nanoparticles. The cytotoxicity of Pt vs. NPs (Pt(Iv)) (A) and (D), NPS (CAD) vs. Dox (B) and (E), Pt + Dox vs. NPs (Pt(IV) + CAD) (C) and (F) to A2780 and A2780DDP.

growth-dependent cyclin-dependent kinase (CDK) activity and promoted the transition of $\mathrm{G}_{1}$ to $\mathrm{S}$ phase. $\mathrm{S}$ phase mainly contributed to the duplication of DNA, and most of the RNA and protein was synthesized during the $\mathrm{G}_{2} / \mathrm{M}$ phase..$^{22}$ As presented in Fig. 6, the free Pt and free Dox led to increased cell cycle arrest in the $S$ phase and $\mathrm{G}_{2} / \mathrm{M}$ phase respectively, which was consistent to previous studies. ${ }^{23,24}$ The $S$ phase arrest of NPs $(\mathrm{Pt}(\mathrm{Iv}))$ was superior to free Pt treatment. On the other hand, the $\mathrm{G}_{2} / \mathrm{M}$ phase arrest of free Dox was stronger than NPs (CAD). As compared to PBS group, we observed that the ratio of cells in both $\mathrm{S}$ phase and $\mathrm{G}$ phase increased significantly for NPs (Pt(Iv) $+\mathrm{CAD}$ ) groups, indicating that they could induce cell apoptosis by interfering with the synthesis of DNA. Correspondingly, in Fig. 7, the cell apoptotic study for A2780DDP was also carried out. The result suggested that the NPs (Pt(Iv) + CAD) were more effective in promoting cell apoptosis than single free drug or single-drug loaded nanoparticles.

Table 2 The summary of $I C_{50}$ and $\mathrm{Cl}$ index. $\star$ represents $I C_{50}$ based on Pt. $\bullet$ represents $I_{50}$ based on Dox. N.A. represents not applicable

\begin{tabular}{llllll}
\hline & $\mathrm{IC}_{50}$ & & & $\mathrm{CI}_{50}$ \\
\cline { 2 - 3 } \cline { 5 - 6 } & $\mathrm{A} 2780$ & A2780DDP & & A2780 & A2780DDP \\
\hline Pt & 9.41 & 31.03 & & N.A. & N.A. \\
Dox & 1.08 & 1.10 & & N.A. & N.A. \\
Pt + Dox & $1.22^{\star} / 0.75^{\star}$ & $0.99^{\star} / 0.61$ & 0.87 & 0.59 \\
NPs (Pt(Iv) + CAD) & $1.38^{\star} / 0.72 \star$ & $0.99^{\star} / 0.60$ & 0.55 & 0.21 \\
NPs (Pt(IV)) & 3.80 & 9.61 & & N.A. & N.A. \\
NPs (CAD) & 3.84 & 5.7 & & N.A. & N.A.
\end{tabular}

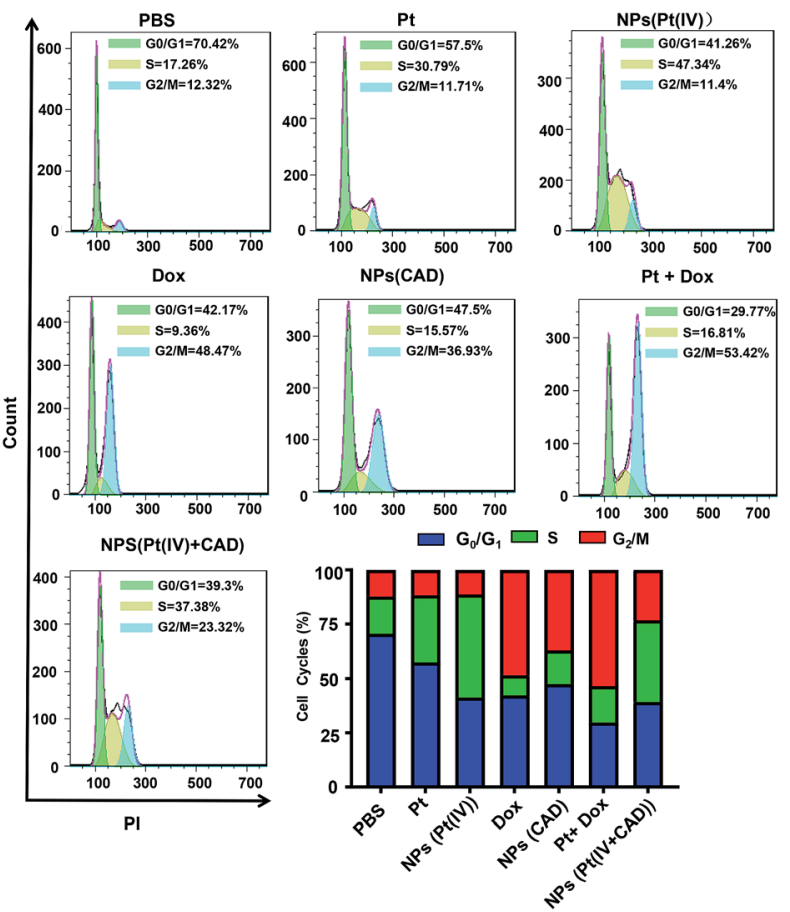

Fig. 6 Cell cycles of free Pt, Dox, and, nanoparticles in A2780DDP. The cell cycle arrest induced by PBS, NPs (Pt(Iv)), Pt, Dox, NPs (CAD), Pt + Dox, NPs (Pt(IV) + CAD).

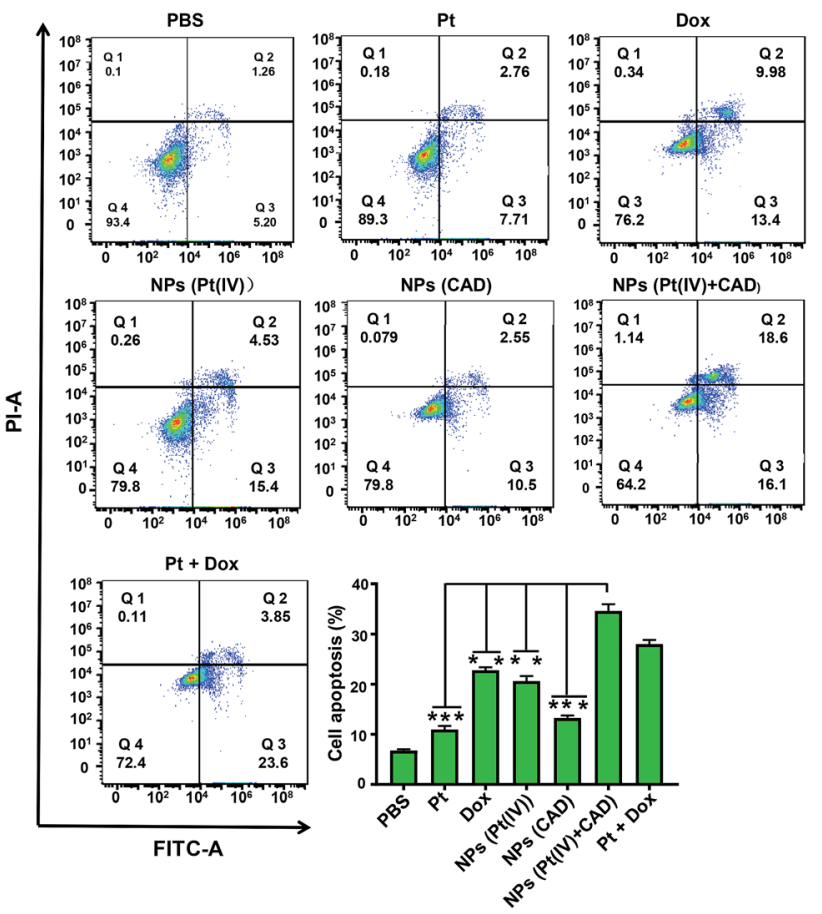

Fig. 7 Cell apoptosis of Pt, Dox, and nanoparticles in A2780DDP. The cell apoptosis induced by NPs (Pt(Iv) + CAD), Pt + Dox, NPs (Pt(Iv)), Cis, NPs (CAD), Dox and PBS. Significance is defined as $* * P<0.001, * P<0.01$.

\section{Conclusion}

$\mathrm{pH} /$ redox-sensitive nanoparticles with dual loaded prodrugs, NPs (Pt(Iv) + CAD), were successfully prepared in this study. The 
hydrophobic segment of $\mathrm{Pt}(\mathrm{IV})$ prodrugs and CAD were not only the core of NPs (Pt(Iv) + CAD), but also remained stable, which resulted in low drug release of both drugs under the neutral condition. However, the NPs (Pt(Iv) + CAD) could efficiently release platinum and Dox under acidic environment with high GSH concentration, which was the main characteristics for tumour cells. Furthermore, great synergistic effect was achieved on the NPs (Pt(Iv) + CAD). Ovarian cancer cells treated with NPs $(\mathrm{Pt}(\mathrm{Iv})+\mathrm{CAD})$ demonstrated increased $\mathrm{S}$ and $\mathrm{G}_{2} / \mathrm{M}$ phase arrest for cell cycles, resulting in the apoptosis of cancer cells. Therefore, a promising and efficient anti-cancer therapy has been achieved with NPs (Pt(Iv) + CAD).

\section{Conflicts of interest}

There are no conflicts to declare.

\section{Notes and references}

1 R. L. Siegel, K. D. Miller and A. Jemal, Ca-Cancer J. Clin., 2019, 69, 7-34.

2 C. B. Tempfer, G. A. Rezniczek, E. Paula, S. Wiebke and R. Marc-André, Anticancer Res., 2015, 35, 6723-6729.

3 C. B. Tempfer, U. Giger-Pabst, V. Seebacher, M. Petersen, A. Dogan and G. A. Rezniczek, Gynecol. Oncol., 2018, 150, 23-30.

4 C. B. Tempfer, I. Celik, W. Solass, B. Buerkle, U. G. Pabst, J. Zieren, D. Strumberg and M. A. Reymond, Gynecol. Oncol., 2014, 132, 307-311.

5 G. Perilongo, R. Maibach, E. Shafford, L. Brugieres, P. Brock, B. Morland, B. de Camargo, J. Zsiros, D. Roebuck, A. Zimmermann, D. Aronson, M. Childs, E. Widing, V. Laithier, J. Plaschkes, J. Pritchard, M. Scopinaro, G. MacKinlay and P. Czauderna, N. Engl. J. Med., 2009, 361, 1662-1670.

6 R. Mezencev, Curr. Cancer Drug Targets, 2015, 14, 794-816.

7 R. Qi, Y. Wang, P. M. Bruno, H. Xiao, Y. Yu, T. Li, S. Lauffer, W. Wei, Q. Chen, X. Kang, H. Song, X. Yang, X. Huang, A. Detappe, U. Matulonis, D. Pepin, M. T. Hemann,
M. J. Birrer and P. P. Ghoroghchian, Nat. Commun., 2017, 8, 2166.

8 Y. Y. Li, L. Yan, Z. Li and H. Q. Dong, $R S C A d v ., 2015,5$, 73752-73759.

9 Z. Zhou, J. Tang, Q. Sun, W. J. Murdoch and Y. Shen, J. Mater. Chem. A, 2015, 3, 7594-7603.

10 H. Xiao, H. Song, Y. Qiang, H. Cai, R. Qi, L. Yan, L. Shi, Y. Zheng, Y. Huang and T. Liu, Biomaterials, 2012, 33, 6507-6519.

11 Z. Yu, Q. Chen, Y. Yang, X. Lin, W. Ma, G. Chen, W. Li and X. Wang, Chem. Commun., 2018, 54, 5369-5372.

12 H. Song, J. Zhang, W. Wang, P. Huang, Y. Zhang, J. Liu, C. Li and D. Kong, Colloids Surf., B, 2015, 136, 365-374.

13 M. L. Patil, Z. Min and M. Tamara, ACS Nano, 2011, 5, 18771887.

14 D. Jizhe, G. Ningning, W. Yanan, Y. Huqiang, F. Shengtao, M. Yifan and C. Lintao, Biomacromolecules, 2012, 13, 37953804.

15 L. Bildstein, C. Dubernet and P. Couvreur, Adv. Drug Delivery Rev., 2011, 63, 3-23.

16 C. Luo, J. Sun, B. Sun and Z. He, Trends Pharmacol. Sci., 2014, 35, 556-566.

17 X. Yang, Y. Yu, X. Huang, Q. Chen, H. Wu, R. Wang, R. Qi, Y. Miao and Y. Qiu, Mater. Sci. Eng., C, 2019, 96, 96-104.

18 A. M. Master and G. A. Sen, Nanomedicine, 2012, 7, 18951906.

19 Y. Yu, Q. Xu, S. He, H. Xiong, Q. Zhang, W. Xu, V. Ricotta, L. Bai, Q. Zhang, Z. Yu, J. Ding, H. Xiao and D. Zhou, Coord. Chem. Rev., 2019, 387, 154-179.

20 W. Xu, J. Ding and X. Chen, Biomacromolecules, 2017, 18(10), 3291-3301.

21 L. Zhao, M. G. Wientjes and J. L. Au, Clin. Cancer Res., 2004, 10, 7994-8004.

22 B. Cosetta, J. M. Skotheim and R. A. M. Bruin De, Nat. Rev. Mol. Cell Biol., 2013, 14, 518-528.

23 W. Li, M. Jiang, Y. Cao, L. Yan, R. Qi, Y. Li and X. Jing, Bioconjugate Chem., 2016, 27, 1802-1806.

24 M. Zhang, S. Jing, Z. Jie, J. Zhang, X. Zang, M. Qiao, X. Zhao, H. Hu and D. W. Chen, J. Mater. Chem. B, 2017, 5, 3970-3981. 